\title{
Construction of positivity-preserving numerical method for stochastic SIVS epidemic model
}

\author{
Wenrui Li ${ }^{1}$ and Qimin Zhang ${ }^{1 *}$
}

\section{${ }^{*}$ Correspondence:}

qiminzhang89@sina.com

'School of Mathematics and

Statistics, Ningxia University,

Yinchuan, P.R. China

\section{Springer}

\begin{abstract}
In this paper we propose the balanced implicit numerical techniques for maintaining the nonnegative path of the solution in stochastic

susceptible-infected-vaccinated-susceptible (SIVS) epidemic model. We can hardly acquire the explicit solution for the SIVS model, so we often use the numerical scheme to produce approximate solutions. The Euler-Maruyama (EM) method is a useful and effective means in producing numerical solutions of SIVS model. The EM method to simulate the stochastic SIVS model often results in the problem that the numerical solution is not positive. In order to eliminate the negative path of the solution in a stochastic SIVS epidemic model, we construct a numerical method preserving positivity for the SIVS model. It is proved that the balanced implicit method (BIM) can preserve positivity and we show the convergence of the BIM numerical approximate solution to the exact solution. Finally, a numerical example is offered to support the theoretical results and verify the availability of the approach.
\end{abstract}

Keywords: Euler-Maruyama scheme; SIVS model; Balanced implicit method; Convergence

\section{Introduction}

Recent global infectious diseases (such as the outbreak of H7N9 influenza in 2013 and Ebola disease in 2014) resulted in a lot of biological deaths and substantial financial ruins. Infectious diseases are a major concern of public. The modeling of infection diseases is extremely important to research the mechanisms of diseases. A mathematical model is considered as an effective way to forecast the outbreak of disease. In particular, stochastic epidemic models have come to play an important role in the control of diseases, which is an extremely significant tool to account for the real world.

As is well known, the SIVS epidemic model is one of the most important models in epidemiology and biomathematics. Many authors have analyzed the susceptible-infectedsusceptible (SIS) epidemic model with vaccination. Shi et al. [1] analyzed the effect of impulsive vaccination on a susceptible-infected-recovered (SIR) epidemic model. In [2], Nie et al. presented the existence and orbital stability of an order- 1 or order- 2 periodic solution for the SIVS model. Liu et al. [3] established two classes of susceptible-vaccinatedinfected-removed models to describe a continuous vaccination strategy and a fixed-time pulse vaccination strategy, respectively. Omondi et al. [4] analyzed a mathematical model

(c) The Author(s) 2019. This article is distributed under the terms of the Creative Commons Attribution 4.0 International License (http://creativecommons.org/licenses/by/4.0/), which permits unrestricted use, distribution, and reproduction in any medium, provided you give appropriate credit to the original author(s) and the source, provide a link to the Creative Commons license, and indicate if changes were made. 
of a rotavirus infection incorporating vaccination. Lin et al. [5] pointed out the stationary distribution of a stochastic SIS epidemic model with vaccination. The classical SIVS model was proposed by Tornatore et al. [6], which has the following form:

$$
\left\{\begin{array}{l}
d S=[(1-p) \mu+\alpha I-(\mu+\psi) S-\beta S I] d t-\sigma S I d w(t), \\
d I=[\beta S I+(1-e) \beta V I-(\mu+\alpha) I] d t+\sigma S I d w(t)+(1-e) \sigma V I d w(t), \\
d V=[p \mu+\psi S-\mu V-(1-e) \beta V I] d t-(1-e) \sigma V I d w(t),
\end{array}\right.
$$

where $S$ denotes the population sizes of susceptible individuals, $I$ denotes the population sizes of infected individuals, $V$ denotes the density of vaccines who have begun the vaccination process. $\mu$ represents the birth rate with susceptible individuals, where a proportion $p$ of them obtained vaccination immediately after birth. Susceptible individuals can either die with rate $\mu$, vaccinated with rate $\psi$ or obtain infected with force of infection $\beta I$ where $\beta$ is the successful transformation rate from infected group to susceptible. Infected individuals can either die with rate $\mu$ or be removed with rate $\alpha$. Vaccinated individuals can either die with rate $\mu$ or acquire infection with force of infection $(1-e) \beta I$ where $e$ measures the efficacy of the vaccine-induced protection against infection. If $e=1$, then the vaccine is perfectly valid in preventing infection, while $e=0$ means that the vaccine has no effect. Here $w(t)$ is a standard Brownian motion with the intensity $\sigma^{2}>0$. All parameters are usually assumed to be positive.

For model (1.1), by [7] and [8] existence and persistence were discussed, respectively. In [9], Yang et al. provided the global threshold dynamics of an SIVS model with waning vaccine-induced immunity and nonlinear incidence. Zhao [10] gave the threshold of a stochastic SIVS epidemic model with nonlinear saturated incidence. Wen et al. in [11] remarked that the threshold of a periodic stochastic SIVS epidemic model with nonlinear incidence. But the stochastic SIVS epidemic model (1.1) rarely has an explicit solution. Therefore, numerical schemes or approximation techniques become the most focus problems in the analysis of stochastic SIVS model is their numerical solution. Up to now, the Euler-Maruyama (EM) scheme is prevalent for stochastic differential equations, which is due to the the simple structure and moderate computational cost. There are many articles investigating this method. In particular, Mao [12] made use of the truncated EulerMaruyama method to discretize the stochastic differential equations. Hu et al. [13] showed a remarkable result of the semi-implicit Euler-Maruyama scheme for stiff stochastic equations. It also should be mentioned that in $[14,15]$ we find the Euler-Maruyama approximation of solutions to stochastic differential equations.

When we use EM scheme for the initial model (1.1), it is crucial that whether the numerical approximate solution is able to converge to the exact solutions. On the other hand, positivity is the most basic trait in many real world systems. For instance, featuring susceptible individuals in the infectious disease modeling is inherently nonnegative. Therefore, preserving the nonnegative path of the exact solution of stochastic SIVS model is also important.

In fact, many numerical methods have been developed to preserve the positivity of the approximate solution [16-18]. Nevertheless, for the preserving positivity numerical solution issue of stochastic epidemic model, to the best of our knowledge, there is not any result. Hence, the main purpose of this present paper is to structure a new method to maintain the nonnegative path of the solution for a stochastic epidemic SIVS model, which is 
the balanced implicit method. The main technique we developed is based on Tan's principle [19].

The main innovation points of this paper are as follows:

(1) Structuring a balanced implicit method to maintain the nonnegative path of the true solution for the stochastic epidemic SIVS model.

(2) The BIM approximate solution will converge to the true solution with order $\frac{1}{2}\left(1-\frac{1}{l}\right)$ for the stochastic SIVS epidemic model.

The arrangement of the paper is as follows. In Sect. 2, we give some necessary notations and preliminaries. Then we define the balanced implicit method. To ensure the positivity of the balanced implicit method, we build feedback controls. In Sect. 3, we show that the balanced implicit method solutions can converge to the true solution. Finally, a numerical example is presented to support the theoretical results and verify the availability of the approach.

\section{Preliminaries and BIM scheme}

\subsection{Necessary notations and preliminaries}

To begin with, we use some notation. Throughout this paper, let $(\Omega, \mathcal{F}, \mathbb{P})$ be a complete probability space with filtration $\left\{\mathcal{F}_{t}\right\}_{t \geq 0}$ satisfying the usual conditions (i.e. it is increasing and right continuous while $\mathcal{F}_{0}$ ) contains all $\mathbb{P}$-null sets, and let $\mathbb{E}$ denote the expectation corresponding to $\mathbb{P}$. Let $w(t)$ be a scalar Brownian motions defined on the space and $T$ be an arbitrary positive number. Moreover, for any $a, b \in R, a \vee b:=\max \{a, b\}$, and $a \wedge$ $b:=\min \{a, b\}$. If $G$ is a set, its indicator function by $1_{G}$, namely $1_{G}(x)=1$ if $x \in G$ and 0 otherwise.

According to model (1.1), we denote the total population $N(t)=S(t)+I(t)+V(t), N(0)=$ $S(0)+I(0)+V(0)$. It is easy to see that

$$
d(S+I+V)=[\mu-\mu(S+I+V)] d t .
$$

Then

$$
\begin{aligned}
S+I+V & =1+e^{-\mu t(S(0)+I(0)+V(0)-1)} \\
& \leq \begin{cases}1, & \text { if } S(0)+I(0)+V(0) \leq 1, \\
S(0)+I(0)+V(0), & \text { if } S(0)+I(0)+V(0)>1,\end{cases} \\
& :=K .
\end{aligned}
$$

Thus the region

$$
\Gamma=\{(S, I, V): S>0, I>0, V>0, S+I+V \leq K\}
$$

is a positively invariant set of model (1.1). Hence from now on, we always assume that the initial value are bounded $(S(0), I(0), V(0)) \in \Gamma$.

Theorem 2.1 System (1.1) has a unique positive solution on $[0, T]$.

Proof The proof of this theorem is similar to that in [20]. 


\subsection{Life time and BIM scheme}

The idea of the life time of of numerical scheme was presented by Schurz in [21]. Schurz employed the notion of an algorithm having eternal lifetime, where we utilized this life time for (1.1) as follows.

Definition 2.1 Assume that the process $S_{t}=S(t), I_{t}=I(t), V_{t}=V(t): t>0$ satisfying model (1.1) has only nonnegative values a.s. provided that $S_{0} \geq 0, I_{0} \geq 0, V_{0} \geq 0$, i.e. we have

$$
\left\{\begin{array}{l}
\mathbb{P}\left(S_{t}>0\right)=1, \\
\mathbb{P}\left(I_{t}>0\right)=1, \quad \forall t>0 . \\
\mathbb{P}\left(V_{t}>0\right)=1,
\end{array}\right.
$$

Then a numerical solution $S_{t_{n}}, I_{t_{n}}, V_{t_{n}}$ possesses an eternal life time if

$$
\left\{\begin{array}{l}
\mathbb{P}\left(S_{n+1} \mid S_{n}>0\right)=1, \\
\mathbb{P}\left(I_{n+1} \mid I_{n}>0\right)=1, \quad \forall n>0 . \\
\mathbb{P}\left(V_{n+1} \mid V_{n}>0\right)=1,
\end{array}\right.
$$

Applying the EM method to model (1.1) yields

$$
\left\{\begin{aligned}
S_{n+1}= & S_{n}+\left[(1-p) \mu+\alpha I_{n}-(\mu+\psi) S_{n}-\beta S_{n} I_{n}\right] \Delta-\sigma S_{n} I_{n} \Delta w_{n} \\
I_{n+1}= & I_{n}+\left[\beta S_{n} I_{n}+(1-e) \beta V_{n} I_{n}-(\mu+\alpha) I_{n}\right] \Delta+\sigma S_{n} I_{n} \Delta w_{n} \\
& +(1-e) \sigma V_{n} I_{n} \Delta w_{n}, \\
V_{n+1}= & V_{n}+\left[p \mu+\psi S_{n}-\mu V_{n}-(1-e) \beta V_{n} I_{n}\right] \Delta-(1-e) \sigma V_{n} I_{n} \Delta w_{n}
\end{aligned}\right.
$$

let $t_{i} \in[0, T], i=1,2, \ldots m$. Let $\Delta=t_{n+1}-t_{n}, \Delta w_{n}=w\left(t_{n+1}\right)-w\left(t_{n}\right)$ denote the increments of the time and Brownian motion, respectively. Let $t_{n}=n \cdot \Delta$, the time increment is $\Delta=$ $\frac{T}{m} \ll 1$.

Lemma 2.1 The EM approximation (2.3) started in $S_{0}>0, I_{0}>0, V_{0}>0$ has a finite life time.

Proof For simplicity, we let

$$
\begin{aligned}
& F_{1}=\left[(1-p) \mu+\alpha I_{n}-(\mu+\psi) S_{n}-\beta S_{n} I_{n}\right], \\
& F_{2}=\left[\beta S_{n} I_{n}+(1-e) \beta V_{n} I_{n}-(\mu+\alpha) I_{n}\right], \\
& F_{3}=\left[p \mu+\psi S_{n}-\mu V_{n}-(1-e) \beta V_{n} I_{n}\right] .
\end{aligned}
$$

It is easy to see that

$$
\begin{aligned}
& \mathbb{P}\left\{S_{n+1}<0\right\}=\mathbb{P}\left\{S_{n}+F_{1} \Delta-\sigma S_{n} I_{n} \Delta w_{n}<0\right\}>0, \\
& \mathbb{P}\left\{I_{n+1}<0\right\}=\mathbb{P}\left\{I_{n}+F_{2} \Delta+\sigma S_{n} I_{n} \Delta w_{n}+(1-e) \sigma V_{n} I_{n} \Delta w_{n}<0\right\}>0, \\
& \mathbb{P}\left\{V_{n+1}<0\right\}=\mathbb{P}\left\{V_{n}+F_{3} \Delta-(1-e) \sigma V_{n} I_{n} \Delta w_{n}<0\right\}>0 .
\end{aligned}
$$


The EM approximation (2.3) fails to preserve positivity, since $\Delta w_{n}$ take all values $c \in \mathbb{R}$ with a positive probability.

Therefore, how it is possible to prevent an approximation integration method from becoming negative. In this paper, we construct the BIM to preserve positivity of rigid SDEs.

The BIM numerical solution to this scheme is defined by

$$
\left\{\begin{aligned}
S_{n+1}^{B}= & S_{n}+\left[(1-p) \mu+\alpha I_{n}-(\mu+\psi) S_{n}-\beta S_{n} I_{n}\right] \Delta-\sigma S_{n} I_{n} \Delta w_{n} \\
& +C_{n}\left(S_{n}-S_{n+1}^{B}\right), \\
I_{n+1}^{B}= & I_{n}+\left[\beta S_{n} I_{n}+(1-e) \beta V_{n} I_{n}-(\mu+\alpha) I_{n}\right] \Delta+\sigma S_{n} I_{n} \Delta w_{n} \\
& +(1-e) \sigma V_{n} I_{n} \Delta w_{n}+D_{n}\left(I_{n}-I_{n+1}^{B}\right), \\
V_{n+1}^{B}= & V_{n}+\left[p \mu+\psi S_{n}-\mu V_{n}-(1-e) \beta V_{n} I_{n}\right] \Delta-(1-e) \sigma V_{n} I_{n} \Delta w_{n} \\
& +E_{n}\left(V_{n}-V_{n+1}^{B}\right),
\end{aligned}\right.
$$

where

$$
\left\{\begin{array}{l}
C_{n}=\left(\mu+\psi+\beta I_{n}\right) \Delta+\sigma I_{n}\left|\Delta w_{n}\right| \\
D_{n}=(\mu+\alpha) \Delta+\sigma\left(S_{n}+V_{n}\right)\left|\Delta w_{n}\right| \\
E_{n}=\left(\mu+\beta I_{n}\right) \Delta+\sigma I_{n}\left|\Delta w_{n}\right|
\end{array}\right.
$$

with initial values $S_{0}^{B}=S_{0}, I_{0}^{B}=I_{0}, V_{0}^{B}=V_{0}$.

Suppose that $S_{n}>0, I_{n}>0$ and $V_{n}>0$, then

$$
\left\{\begin{array}{l}
0<\left(1+\left(\mu+\psi+\beta I_{n}\right) \Delta+\sigma I_{n}\left|\Delta w_{n}\right|\right)^{-1}<M_{1} \\
0<\left(1+(\mu+\alpha) \Delta+\sigma\left(S_{n}+V_{n}\right)\left|\Delta w_{n}\right|\right)^{-1}<M_{2} \\
0<\left(1+\left(\mu+\beta I_{n}\right) \Delta+\sigma I_{n}\left|\Delta w_{n}\right|\right)^{-1}<M_{3}
\end{array}\right.
$$

let $M=M_{1} \vee M_{2} \vee M_{3}$, where $M_{1}, M_{2}, M_{3}$ and $M$ are positive constant.

Now we give the following theorem for the positivity of the BIM.

Theorem 2.2 The balanced numerical method (2.6) has an eternal life time.

Proof Under the condition (2.8), the scheme of BIM (2.3) can be rewritten

$$
\left\{\begin{aligned}
S_{n+1}^{B}= & \left(1+C_{n}\right)^{-1}\left[S_{n}+\left((1-p) \mu+\alpha I_{n}-(\mu+\psi) S_{n}-\beta S_{n} I_{n}+\mu S_{n}+\varphi S_{n}\right.\right. \\
& \left.\left.+\beta S_{n} I_{n}\right) \Delta-\sigma S_{n} I_{n} \Delta W_{n}+\sigma S_{n} I_{n}\left|\Delta w_{n}\right|\right], \\
I_{n+1}^{B}= & \left(1+D_{n}\right)^{-1}\left[I_{n}+\left(\beta S_{n} I_{n}+(1-e) \beta V_{n} I_{n}-(\mu+\alpha) I_{n}+\mu I_{n}+\alpha I_{n}\right) \Delta\right. \\
& \left.+\sigma S_{n} I_{n} \Delta w_{n}+(1-e) \sigma V_{n} I_{n} \Delta w_{n}+\sigma\left(S_{n}+V_{n}\right) I_{n}\left|\Delta w_{n}\right|\right], \\
V_{n+1}^{B}= & \left(1+E_{n}\right)^{-1}\left[V_{n}+\left(p \mu+\psi S_{n}-\mu V_{n}-(1-e) \beta V_{n} I_{n}+\mu V_{n}+\beta V_{n} I_{n}\right) \Delta\right. \\
& \left.-(1-e) \sigma V_{n} I_{n} \Delta w_{n}+\sigma I_{n} V_{n}\left|\Delta w_{n}\right|\right] .
\end{aligned}\right.
$$


We can easily see that

$$
\begin{aligned}
& S_{n+1}^{B} \geq\left(1+C_{n}\right)^{-1}\left(S_{n}+\left((1-p) \mu+\alpha I_{n}\right) \Delta\right) \geq 0, \\
& I_{n+1}^{B} \geq\left(1+D_{n}\right)^{-1}\left(I_{n}+\left(\beta S_{n} I_{n}+(1-e) \beta V_{n} I_{n}\right) \Delta\right) \geq 0, \\
& V_{n+1}^{B} \geq\left(1+E_{n}\right)^{-1}\left(V_{n}+\left(p \mu+\psi S_{n}+e \beta V_{n} I_{n}\right) \Delta\right) \geq 0 .
\end{aligned}
$$

This completes the proof.

\section{Convergence of the balanced method}

In this section, we show the main results for the strong convergence of the balanced method.

For model (2.3), when $t \in\left[t_{n}, t_{n+1}\right)$, we define the continuous approximation

$$
\left\{\begin{aligned}
S_{t}^{E}= & S_{0}+\left[(1-p) \mu+\alpha I_{n}-(\mu+\psi) S_{n}-\beta S_{n} I_{n}\right]\left(t-t_{n}\right)-\sigma S_{n} I_{n}\left(w_{t}-w_{t_{n}}\right), \\
I_{t}^{E}= & I_{0}+\left[\beta S_{n} I_{n}+(1-e) \beta V_{n} I_{n}-(\mu+\alpha) I_{k}\right]\left(t-t_{n}\right)+\sigma S_{n} I_{n}\left(w_{t}-w_{t_{n}}\right) \\
& +(1-e) \sigma V_{n} I_{n}\left(w_{t}-w_{t_{n}}\right), \\
V_{t}^{E}= & V_{0}+\left[p \mu+\psi S_{n}-\mu V_{n}-(1-e) \beta V_{n} I_{n}\right]\left(t-t_{n}\right)-(1-e) \sigma V_{n} I_{n}\left(w_{t}-w_{t_{n}}\right) .
\end{aligned}\right.
$$

Hence, (3.1) can be rewritten in the integral form

$$
\left\{\begin{aligned}
S_{t}^{E}= & S_{0}+\int_{0}^{t}\left[(1-p) \mu+\alpha \bar{I}_{s}-(\mu+\psi) \bar{S}_{s}-\beta \bar{S}_{s} \bar{I}_{s}\right] d s-\sigma \bar{S}_{s} \bar{I}_{s} d w(s), \\
I_{t}^{E}= & I_{0}+\int_{0}^{t}\left[\beta \bar{S}_{s} \bar{I}_{s}+(1-e) \beta \bar{V}_{s} \bar{I}_{s}-(\mu+\alpha) \bar{I}_{s}\right] d s+\int_{0}^{t} \sigma \bar{S}_{s} \bar{I}_{s} d w(s) \\
& +\int_{0}^{t}(1-e) \sigma \bar{V}_{s} \bar{I}_{s} d w(s), \\
V_{t}^{E}= & V_{0}+\int_{0}^{t}\left[p \mu+\psi \bar{S}_{s}-\mu \bar{V}_{s}-(1-e) \beta \bar{V}_{s} \bar{I}_{s}\right] d s-\int_{0}^{t}(1-e) \sigma \bar{V}_{s} \bar{I}_{s} d w(s),
\end{aligned}\right.
$$

where $\bar{S}(t)=\sum_{n=0}^{\left[\frac{T}{\Delta}\right]} S_{n} 1_{\left[t_{n}, t_{n+1}\right)}(t), \bar{I}(t)=\sum_{n=0}^{\left[\frac{T}{\Delta}\right]} I_{n} 1_{\left[t_{n}, t_{n+1}\right)}(t)$, and $\bar{V}(t)=\sum_{n=0}^{\left[\frac{T}{\Delta}\right]} V_{n} 1_{\left[t_{n}, t_{n+1}\right)}(t)$ are step processes.

To prove the convergence theorem, we first need to consider the following lemma, which reveals that the continuous EM solution $S_{t}^{E}, I_{t}^{E}, V_{t}^{E}$ will converge to the step process $\bar{S}_{t}, \bar{I}_{t}$, $\bar{V}_{t}$. Since the drift and diffusion coefficients of model (1.1) do not satisfy the linear growth condition, the traditional theory of convergence is not applicable for model (1.1). To tackle this problem, we introduce a stopping time.

Lemma 3.1 For any positive number $K$, define the stopping time [22] $q=\rho_{K} \wedge \gamma_{K} \wedge T$, where $\rho_{K}=\inf \left\{t \in[0, T] ;\{S(t), I(t), V(t)\} \notin\left[\frac{1}{K}, K\right]\right\}$ and $\gamma_{K}=\inf \{t \in[0, T] ;\{\bar{S}(t), \bar{I}(t), \bar{V}(t)\} \notin$ $\left.\left[\frac{1}{K}, K\right]\right\}$. Then, for any integer $l \geq 2$, there exists a constant $C_{1}$ independent of $\Delta$ such that

$$
\begin{aligned}
& \mathbb{E}\left(\sup _{0 \leq t \leq T}\left[\left(S_{t}^{E}(t \wedge q)-\bar{S}(t \wedge q)\right)^{2}+\left(I_{t}^{E}(t \wedge q)-\bar{I}(t \wedge q)\right)^{2}+\left(V_{t}^{E}(t \wedge q)-\bar{V}(t \wedge q)\right)^{2}\right]\right) \\
& \quad \leq C_{1} \Delta^{1-\frac{1}{l}}
\end{aligned}
$$

Proof For $t \in[0, T]$, let $\left[\frac{t}{\Delta}\right]$ be the integer part of $\frac{t}{\Delta}$. For simplicity, we show that the approximate solution $S_{t}^{E}, I_{t}^{E}, V_{t}^{E}$ are close to $\bar{S}_{t}, \bar{I}_{t}, \bar{V}_{t}$, respectively. 
First, we have

$$
\begin{aligned}
S_{t}^{E}(t \wedge q)-\bar{S}(t \wedge q)= & \int_{\left[\frac{t \wedge q}{\Delta}\right] \Delta}^{t \wedge q}[(1-p) \mu+\alpha \bar{I}(u)-(\mu+\psi) \bar{S}(u)-\beta \bar{S}(u) \bar{I}(u)] d u \\
& -\int_{\left[\frac{t \wedge q}{\Delta}\right] \Delta}^{t \wedge q} \sigma \bar{S}(u) \bar{I}(u) d w(u),
\end{aligned}
$$

which gives

$$
\begin{aligned}
{\left[S_{t}^{E}(t\right.} & \wedge q)-\bar{S}(t \wedge q)]^{2} \\
\leq & 2\left(\int_{\left[\frac{t \wedge q}{\Delta}\right] \Delta}^{t \wedge q}[(1-p) \mu+\alpha \bar{I}(u)-(\mu+\psi) \bar{S}(u)-\beta \bar{S}(u) \bar{I}(u)] d u\right)^{2} \\
& +2\left(\int_{\left[\frac{t \wedge q}{\Delta}\right] \Delta}^{t \wedge q} \sigma \bar{S}(u) \bar{I}(u) d w(u)\right)^{2} \\
\leq & 2 \int_{\left[\frac{t \wedge q}{\Delta}\right] \Delta}^{t \wedge q} 1 d u \int_{\left[\frac{t \wedge q}{\Delta}\right] \Delta}^{t \wedge q}[(1-p) \mu+\alpha \bar{I}(u)-(\mu+\psi) \bar{S}(u)-\beta \bar{S}(u) \bar{I}(u)]^{2} d u \\
& +2 \sigma^{2} K^{4}\left[w(t \wedge q)-w\left(\left[\frac{t \wedge q}{\Delta}\right] \Delta\right)\right]^{2} \\
\leq & 2 \Delta \int_{\left[\frac{t \wedge q}{\Delta}\right] \Delta}^{t \wedge q}[(1-p) \mu+\alpha \bar{I}(u)-(\mu+\psi) \bar{S}(u)-\beta \bar{S}(u) \bar{I}(u)]^{2} d u \\
& +2 \sigma^{2} K^{4}\left[w(t \wedge q)-w\left(\left[\frac{t \wedge q}{\Delta}\right] \Delta\right)\right]^{2} \\
\leq & 8\left[(1-p)^{2} \mu^{2}+\alpha^{2} K^{2}+(\mu+\psi)^{2} K^{2}+\beta^{2} K^{4}\right] \Delta^{2} \\
& +2 \sigma^{2} K^{4}\left[w(t \wedge q)-w\left(\left[\frac{t \wedge q}{\Delta}\right] \Delta\right)\right]^{2} .
\end{aligned}
$$

We hence have

$$
\begin{aligned}
\mathbb{E}\left(\sup _{0 \leq t \leq T}\left[S_{t}^{E}(t \wedge q)-\bar{S}(t \wedge q)\right]^{2}\right) \\
\leq 8\left[(1-p)^{2} \mu^{2}+\alpha^{2} K^{2}+(\mu+\psi)^{2} K^{2}+\beta^{2} K^{4}\right] \Delta^{2} \\
\quad+2 \sigma^{2} K^{4} \mathbb{E}\left(\sup _{0 \leq t \leq T \wedge q}\left[w(t \wedge q)-w\left(\left[\frac{t \wedge q}{\Delta}\right] \Delta\right)\right]^{2}\right) \\
\leq 8\left[(1-p)^{2} \mu^{2}+\alpha^{2} K^{2}+(\mu+\psi)^{2} K^{2}+\beta^{2} K^{4}\right] \Delta^{2} \\
\quad+2 \sigma^{2} K^{4} \mathbb{E}\left(\sup _{0 \leq t \leq T}\left[w(t \wedge q)-w\left(\left[\frac{t \wedge q}{\Delta}\right] \Delta\right)\right]^{2}\right) .
\end{aligned}
$$

Next, we have

$$
\begin{aligned}
& I_{t}^{E}(t \wedge q)-\bar{I}(t \wedge q) \\
& \quad=\int_{\left[\frac{t \wedge q}{\Delta}\right] \Delta}^{t \wedge q}[\beta \bar{S}(u) \bar{I}(u)+(1-e) \beta \bar{V}(u) \bar{I}(u)-(\mu+\alpha) \bar{I}(u)] d u
\end{aligned}
$$




$$
+\int_{\left[\frac{t \wedge q}{\Delta}\right] \Delta}^{t \wedge q}(1-e) \sigma \bar{V}(u) \bar{I}(u) d w(u)+\int_{\left[\frac{t \wedge q}{\Delta}\right] \Delta}^{t \wedge q} \sigma \bar{S}(u) \bar{I}(u) d w(u),
$$

which gives

$$
\begin{aligned}
{\left[I_{t}^{E}(t\right.} & \wedge q)-\bar{I}(t \wedge q)]^{2} \\
\leq & 3\left(\int_{\left[\frac{t \wedge q}{\Delta}\right] \Delta}^{t \wedge q}[\beta \bar{S}(u) \bar{I}(u)+(1-e) \beta \bar{V}(u) \bar{I}(u)-(\mu+\alpha) \bar{I}(u)] d u\right)^{2} \\
& +3(1-e)^{2} \sigma^{2}\left(\int_{\left[\frac{t \wedge q}{\Delta}\right] \Delta}^{t \wedge q} \bar{V}(u) \bar{I}(u) d w(u)\right)^{2}+3 \sigma^{2}\left(\int_{\left[\frac{t \wedge q}{\Delta}\right] \Delta}^{t \wedge q} \bar{S}(u) \bar{I}(u) d w(u)\right)^{2} \\
\leq & 9\left[\beta^{2} K^{4}+(1-e)^{2} \beta^{2} K^{4}+(\mu+\psi)^{2} K^{2}\right] \Delta^{2} \\
& +3\left[(1-e) 2 \sigma^{2} K^{4}+3 \sigma^{2} K^{4}\right]\left[w(t \wedge q)-w\left(\left[\frac{t \wedge q}{\Delta}\right] \Delta\right)\right]^{2} .
\end{aligned}
$$

We hence have

$$
\begin{aligned}
& \mathbb{E}\left(\sup _{0 \leq t \leq T}\left[I_{t}^{E}(t \wedge q)-\bar{I}(t \wedge q)\right]^{2}\right) \\
& \leq 9\left[\beta^{2} K^{4}+(1-e)^{2} \beta^{2} K^{4}+(\mu+\psi)^{2} K^{2}\right] \Delta^{2} \\
&+3\left[(1-e) 2 \sigma^{2} K^{4}+3 \sigma^{2} K^{4}\right] \mathbb{E}\left(\sup _{0 \leq t \leq T \wedge q}\left[w(t \wedge q)-w\left(\left[\frac{t \wedge q}{\Delta}\right] \Delta\right)\right]^{2}\right) \\
& \leq 9\left[\beta^{2} K^{4}+(1-e)^{2} \beta^{2} K^{4}+(\mu+\psi)^{2} K^{2}\right] \Delta^{2} \\
&+3\left[(1-e) 2 \sigma^{2} K^{4}+3 \sigma^{2} K^{4}\right] \mathbb{E}\left(\sup _{0 \leq t \leq T}\left[w(t \wedge q)-w\left(\left[\frac{t \wedge q}{\Delta}\right] \Delta\right)\right]^{2}\right) .
\end{aligned}
$$

Then we have

$$
\begin{aligned}
V_{t}^{E}(t \wedge q)-\bar{V}(t \wedge q)= & \int_{\left[\frac{t \wedge q}{\Delta}\right] \Delta}^{t \wedge q}[p \mu+\psi \bar{S}(u)-\mu \bar{V}(u)-(1-e) \beta \bar{V}(u) \bar{I}(u)] d u \\
& +\int_{\left[\frac{t \wedge q}{\Delta}\right] \Delta}^{t \wedge q}(1-e) \sigma|\bar{V}(u)||\bar{I}(u)| d w(u),
\end{aligned}
$$

which gives

$$
\begin{aligned}
{\left[V_{t}^{E}(t \wedge q)-\bar{V}(t \wedge q)\right]^{2} \leq } & 8\left(p^{2} \mu^{2}+\psi^{2} K^{2}+(1-e)^{2} \beta^{2} K^{4}\right) \Delta^{2} \\
& +2(1-e)^{2} \sigma^{2} K^{4}\left[w(t \wedge q)-w\left(\left[\frac{t \wedge q}{\Delta}\right] \Delta\right)\right]^{2}
\end{aligned}
$$

We hence have

$$
\begin{aligned}
\mathbb{E}\left(\sup _{0 \leq t \leq T}\left[V_{t}^{E}(t \wedge q)-\bar{V}(t \wedge q)\right]^{2}\right) \\
\leq 8\left[p^{2} \mu^{2}+\psi^{2} K^{2}+(1-e)^{2} \beta^{2} K^{4}\right] \Delta^{2} \\
\quad+2(1-e)^{2} \sigma^{2} K^{4} \mathbb{E}\left(\sup _{0 \leq t \leq T}\left[w(t \wedge q)-w\left(\left[\frac{t \wedge q}{\Delta}\right] \Delta\right)\right]^{2}\right) .
\end{aligned}
$$


By the Hölder inequality,

$$
\begin{aligned}
& \mathbb{E}\left(\sup _{0 \leq t \leq T}\left[w(t \wedge q)-w\left(\left[\frac{t \wedge q}{\Delta}\right] \Delta\right)\right]^{2}\right) \\
& \quad \leq\left(\mathbb{E}\left(\sup _{0 \leq t \leq T}\left[w(t \wedge q)-w\left(\left[\frac{t \wedge q}{\Delta}\right] \Delta\right)\right]^{2 l}\right)\right)^{\frac{1}{l}} .
\end{aligned}
$$

By the Doob martingale inequality, we get

$$
\begin{aligned}
\mathbb{E}\left(\sup _{0 \leq t \leq T}\left[w(t \wedge q)-w\left(\left[\frac{t \wedge q}{\Delta}\right] \Delta\right)\right]^{2 l}\right) & \leq \mathbb{E}\left(\sup _{0 \leq k \leq\left[\frac{T}{\Delta}\right]} \sup _{k \Delta \leq r \leq \Delta(k+1)}[w(r)-w(\Delta k)]^{2 l}\right) \\
& \leq \sum_{k=0}^{\left[\frac{T}{\Delta}\right]} \mathbb{E}\left[\sup _{k \Delta \leq r \leq \Delta(k+1)}|w(r)-w(\Delta k)|^{2 l}\right] \\
& \leq\left(\frac{2 l}{2 l-1}\right) \sum_{k=0}^{\left[\frac{T}{\Delta}\right]} \mathbb{E}|w(\Delta(k+1))-w(\Delta k)|^{2 l} \\
& \leq\left(\frac{2 l}{2 l-1}\right)^{2 l} \sum_{k=0}^{\left[\frac{T}{\Delta}\right]}(2 l-1) ! ! \Delta^{l} \\
& \leq\left(\frac{2 l}{2 l-1}\right)^{2 l}(2 l-1) ! ! \Delta^{l-1}(T+1),
\end{aligned}
$$

therefore, we have

$$
\begin{gathered}
\mathbb{E}\left(\sup _{0 \leq t \leq T}\left[w(t \wedge q)-w\left(\left[\frac{t \wedge q}{\Delta}\right] \Delta\right)\right]^{2}\right) \\
\leq\left(\left(\frac{2 l}{2 l-1}\right)^{2 l}(2 l-1) ! ! \Delta^{l-1}(T+1)\right)^{\frac{1}{l}} \\
\leq\left(\frac{2 l}{2 l-1}\right)^{2}(2 l-1) ! !^{\frac{1}{l}} \Delta^{1-\frac{1}{l}}(T+1)^{\frac{1}{l}}
\end{gathered}
$$

From (3.4) to (3.14), we obtain

$$
\begin{aligned}
& \mathbb{E}\left(\sup _{0 \leq t \leq T}\left[\left[S_{t}^{E}(t \wedge q)-\bar{S}(t \wedge q)\right]^{2}+\left[I_{t}^{E}(t \wedge q)-\bar{I}(t \wedge q)\right]^{2}+\left[V_{t}^{E}(t \wedge q)-\bar{V}(t \wedge q)\right]^{2}\right]\right) \\
& \leq 8\left[(1-p)^{2} \mu^{2}+\alpha^{2} K^{2}+(\mu+\psi)^{2} K^{2}+\beta^{2} K^{4}\right] \Delta^{2} \\
&+2 \sigma^{2} K^{4} \mathbb{E}\left(\sup _{0 \leq t \leq T}\left[w(t \wedge q)-w\left(\left[\frac{t \wedge q}{\Delta}\right] \Delta\right)\right]^{2}\right) \\
&+9\left[\beta^{2} K^{4}+(1-e)^{2} \beta^{2} K^{4}+(\mu+\psi)^{2} K^{2}\right] \Delta^{2} \\
&+3\left[(1-e) 2 \sigma^{2} K^{4}+3 \sigma^{2} K^{4}\right] \mathbb{E}\left(\sup _{0 \leq t \leq T}\left[w(t \wedge q)-w\left(\left[\frac{t \wedge q}{\Delta}\right] \Delta\right)\right]^{2}\right) \\
&+\left[p^{2} \mu^{2}+\psi^{2} K^{2}+(1-e)^{2} \beta^{2} K^{4}\right] \Delta^{2}
\end{aligned}
$$




$$
\begin{aligned}
& +2(1-e)^{2} \sigma^{2} K^{4} \mathbb{E}\left(\sup _{0 \leq t \leq T}\left[w(t \wedge q)-w\left(\left[\frac{t \wedge q}{\Delta}\right] \Delta\right)\right]^{2}\right) \\
\leq & 8\left[(1-p)^{2} \mu^{2}+\alpha^{2} K^{2}+(\mu+\psi)^{2} K^{2}+\beta^{2} K^{4}\right] \Delta^{2} \\
& +9\left[\beta^{2} K^{4}+(1-e)^{2} \beta^{2} K^{4}+(\mu+\psi)^{2} K^{2}\right] \Delta^{2} \\
& +\left[p^{2} \mu^{2}+\psi^{2} K^{2}+(1-e)^{2} \beta^{2} K^{4}\right] \Delta^{2}+\left[2 \sigma^{2} K^{4}\right. \\
& \left.+3\left[(1-e) 2 \sigma^{2} K^{4}+3 \sigma^{2} K^{4}\right]+2(1-e)^{2} \sigma^{2} K^{4}\right]\left(\frac{2 l}{2 l-1}\right)^{2}(2 l-1) ! ! \frac{1}{l} \Delta^{1-\frac{1}{l}}(T+1)^{\frac{1}{l}} \\
\leq & C_{1} \Delta^{1-\frac{1}{l}} .
\end{aligned}
$$

The proof of the lemma is complete.

Now we estimate the main result, that is to say, the convergence of the EM approximate solution $S_{t}^{E}, I_{t}^{E}, V_{t}^{E}$ to the solution $S(t), I(t), V(t)$.

Theorem 3.1 Let $S(t), I(t), V(t)$ be the true solution and $S_{t}^{E}, I_{t}^{E}$, $V_{t}^{E}$ be the continuous $E M$ approximate solution to the model (1.1). For any integer $l \geq 2$, there exists a constant $C_{2}$ independent of $\Delta$ such that

$$
\begin{aligned}
& \mathbb{E}\left(\sup _{0 \leq t \leq T}\left[\left(S(t \wedge q)-S_{t}^{E}(t \wedge q)\right)^{2}+\left(I(t \wedge q)-I_{t}^{E}(t \wedge q)\right)^{2}+\left(V(t \wedge q)-V_{t}^{E}(t \wedge q)\right)^{2}\right]\right) \\
& \quad \leq C_{2} \Delta^{1-\frac{1}{l}}
\end{aligned}
$$

Proof For any $0 \leq t \leq T$, we adopt similar method. First, we clearly have

$$
\begin{aligned}
& {\left[S(t \wedge q)-S_{t}^{E}(t \wedge q)\right]^{2}} \\
& \quad \leq 2\left[\int_{0}^{t \wedge q}[\alpha(I(u)-\bar{I}(u))-(\mu+\psi)(S(u)-\bar{S}(u))-\beta(S(u) I(u)-\bar{S}(u) \bar{I}(u))] d u\right]^{2} \\
& \quad+2 \sigma^{2}\left[\int_{0}^{t \wedge q}(S(u) I(u)-\bar{S}(u) \bar{I}(u)) d w(u)\right]^{2}
\end{aligned}
$$

for any $t_{1} \in[0, T]$, using the Hölder inequality and the Doob martingale inequality, we then have

$$
\begin{aligned}
\mathbb{E}\left(\sup _{0 \leq t \leq t_{1}}\left[S(t \wedge q)-S_{t}^{E}(t \wedge q)\right]^{2}\right) \\
\leq 2 T \mathbb{E} \int_{0}^{t_{1} \wedge q}[\alpha(I(u)-\bar{I}(u))-(\mu+\psi)(S(u)-\bar{S}(u))-\beta(S(u) I(u)-\bar{S}(u) \bar{I}(u))]^{2} d u \\
\quad+2 \sigma^{2} \mathbb{E}\left(\sup _{0 \leq t \leq t_{1}}\left[\int_{0}^{t \wedge q}(S(u) I(u)-\bar{S}(u) \bar{I}(u)) d w(u)\right]^{2}\right) \\
\leq 6 \alpha^{2} T \mathbb{E} \int_{0}^{t_{1} \wedge q}(I(u)-\bar{I}(u))^{2} d u+6(\mu+\psi)^{2} T \mathbb{E} \int_{0}^{t_{1} \wedge q}(S(u)-\bar{S}(u))^{2} d u \\
\quad+6 \beta^{2} T \mathbb{E} \int_{0}^{t_{1} \wedge q}(S(u) I(u)-\bar{S}(u) \bar{I}(u))^{2} d u+8 \sigma^{2} \mathbb{E} \int_{0}^{t_{1} \wedge q}(S(u) I(u)-\bar{S}(u) \bar{I}(u))^{2} d u \\
\leq 6 \alpha^{2} T \mathbb{E} \int_{0}^{t_{1} \wedge q}(I(u)-\bar{I}(u))^{2} d u+6(\mu+\psi)^{2} T \mathbb{E} \int_{0}^{t_{1} \wedge q}(S(u)-\bar{S}(u))^{2} d u
\end{aligned}
$$




$$
\begin{aligned}
& +2\left(6 \beta^{2} T+8 \sigma^{2}\right)\left[\mathbb{E} \int_{0}^{t_{1} \wedge q} I(u)^{2}(S(u)-\bar{S}(u))^{2}+\mathbb{E} \int_{0}^{t_{1} \wedge q} \bar{S}(u)^{2}(I(u)-\bar{I}(u))^{2} d u\right] \\
\leq & f_{1} \mathbb{E} \int_{0}^{t_{1} \wedge q}(I(u)-\bar{I}(u))^{2} d u+f_{2} \mathbb{E} \int_{0}^{t_{1} \wedge q}(S(u)-\bar{S}(u))^{2} d u \\
\leq & 2 f_{1}\left[\mathbb{E} \int_{0}^{t_{1} \wedge q}\left(I(u)-I_{t}^{E}(u)\right)^{2} d u+\mathbb{E} \int_{0}^{t_{1} \wedge q}\left(I_{t}^{E}(u)-\bar{I}(u)\right)^{2} d u\right] \\
& +2 f_{2}\left[\mathbb{E} \int_{0}^{t_{1} \wedge q}\left(S(u)-S_{t}^{E}(u)\right)^{2} d u+\mathbb{E} \int_{0}^{t_{1} \wedge q}\left(S_{t}^{E}(u)-\bar{S}(u)\right)^{2} d u\right],
\end{aligned}
$$

where $f_{1}=6 \alpha^{2} T+12 \beta^{2} K^{2} T+16 \sigma^{2} K^{2}, f_{2}=6(\mu+\psi)^{2} T+12 \beta^{2} K^{2} T+16 \sigma^{2} K^{2}$.

Similarly, we have

$$
\begin{aligned}
{[I(t} & \left.\wedge q)-I_{t}^{E}(t \wedge q)\right]^{2} \\
\leq & 3\left[\int_{0}^{t \wedge q}[\beta(S(u) I(u)-\bar{S}(u) \bar{I}(u))+(1-e)(\beta(V(u) I(u)-\bar{V}(u) \bar{I}(u))\right. \\
& -(\mu+\alpha)(I(u)-\bar{I}(u))] d u]^{2}+3(1-e)^{2} \sigma^{2}\left[\int_{0}^{t \wedge q}(V(u) I(u)-\bar{V}(u) \bar{I}(u)) d w(u)\right]^{2} \\
& +3 \sigma^{2}\left[\int_{0}^{t \wedge q}(S(u) I(u)-\bar{S}(u) \bar{I}(u)) d w(u)\right]^{2}
\end{aligned}
$$

we then compute

$$
\begin{aligned}
& \mathbb{E}\left(\sup _{0 \leq t \leq t_{1}}\left[I(t \wedge q)-I_{t}^{E}(t \wedge q)\right]^{2}\right) \\
& \leq 9 T \mathbb{E} \int_{0}^{t \wedge q}\left[\beta^{2}(S(u) I(u)-\bar{S}(u) \bar{I}(u))^{2}+(1-e)^{2} \beta^{2}(V(u) I(u)-\bar{V}(u) \bar{I}(u))^{2}\right. \\
&\left.+(\mu+\alpha)^{2}(I(u)-\bar{I}(u))^{2}\right] d u+12(1-e)^{2} \sigma^{2} \mathbb{E} \int_{0}^{t \wedge q}(V(u) I(u)-\bar{V}(u) \bar{I}(u))^{2} d u \\
&+12 \sigma^{2} \mathbb{E} \int_{0}^{t \wedge q}(S(u) I(u)-\bar{S}(u) \bar{I}(u))^{2} d u \\
& \leq\left(9 \beta^{2} T+12 \sigma^{2}\right) \mathbb{E} \int_{0}^{t \wedge q}(S(u) I(u)-\bar{S}(u) \bar{I}(u))^{2} d u \\
&+9(\mu+\alpha)^{2} T \mathbb{E} \int_{0}^{t \wedge q}(I(u)-\bar{I}(u))^{2} d u \\
&+\left[9(1-e)^{2} \beta^{2} T+12(1-e)^{2} \sigma^{2}\right] \mathbb{E} \int_{0}^{t \wedge q}(V(u) I(u)-\bar{V}(u) \bar{I}(u))^{2} d u \\
& \leq\left(18 \beta^{2} T+24 \sigma^{2}\right)\left[\mathbb{E} \int_{0}^{t \wedge q}(S(u) I(u)-\bar{S}(u) I(u))^{2}+\mathbb{E} \int_{0}^{t \wedge q}(\bar{S}(u) I(u)-\bar{S}(u) \bar{I}(u))^{2}\right] \\
&+\left[18(1-e)^{2} \beta^{2} T+24(1-e)^{2} \sigma^{2}\right]\left[\mathbb{E} \int_{0}^{t \wedge q}(V(u) I(u)-\bar{V}(u) I(u))^{2} d u\right. \\
&\left.+\mathbb{E} \int_{0}^{t \wedge q}(\bar{V}(u) I(u)-\bar{V}(u) \bar{I}(u))^{2} d u\right]+9(\mu+\alpha)^{2} T \mathbb{E} \int_{0}^{t \wedge q}(I(u)-\bar{I}(u))^{2} d u \\
& \leq g \mathbb{E} \int_{0}^{t \wedge q}(S(u)-\bar{S}(u))^{2} d u+g_{2} \mathbb{E} \int_{0}^{t \wedge q}(V(u)-\bar{V}(u))^{2} d u
\end{aligned}
$$




$$
\begin{aligned}
& +g_{3} \mathbb{E} \int_{0}^{t \wedge q}(I(u)-\bar{I}(u))^{2} d u \\
\leq & 2 g_{1}\left[\mathbb{E} \int_{0}^{t \wedge q}\left(S(u)-S_{t}^{E}(u)\right)^{2} d u+\mathbb{E} \int_{0}^{t \wedge q}\left(S_{t}^{E}(u)-\bar{S}(u)\right)^{2} d u\right] \\
& +2 g_{2}\left[\mathbb{E} \int_{0}^{t \wedge q}\left(V(u)-V_{t}^{E}(u)\right)^{2} d u+\mathbb{E} \int_{0}^{t \wedge q}\left(V_{t}^{E}(u)-\bar{V}(u)\right)^{2} d u\right] \\
& +2 g_{3}\left[\mathbb{E} \int_{0}^{t \wedge q}\left(I(u)-I_{t}^{E}(u)\right)^{2} d u+\mathbb{E} \int_{0}^{t \wedge q}\left(I_{t}^{E}(u)-\bar{I}(u)\right)^{2} d u\right]
\end{aligned}
$$

where $g_{1}=\left(18 \beta^{2} T+24 \sigma^{2}\right) K^{2}, g_{2}=\left[(1-e)^{2}\left(18 \beta^{2} T+24 \sigma^{2}\right)\right] K^{2}, g_{3}=\left(18 \beta^{2} T+24 \sigma^{2}\right) K^{2}+$ $18(1-e)^{2} \beta^{2} T K^{2}+24(1-e)^{2} \sigma^{2} K^{2}+9(\mu+\alpha)^{2} T$.

Moreover, we have

$$
\begin{aligned}
& {\left[V(t \wedge q)-V_{t}^{E}(t \wedge q)\right]^{2}} \\
& \leq 2\left[\int_{0}^{t \wedge q}[\psi(S(u)-\bar{S}(u))-\mu(V(u)-\bar{V}(u))\right. \\
& \quad-(1-e) \beta(V(u) I(u)-\bar{V}(u) \bar{I}(u))] d u]^{2} \\
& \quad+2(1-e)^{2} \sigma^{2}\left[\int_{0}^{t \wedge q}(V(u) I(u)-\bar{V}(u) \bar{I}(u)) d w(u)\right]^{2},
\end{aligned}
$$

and we compute that

$$
\begin{aligned}
& \mathbb{E}\left(\sup _{0 \leq t \leq t_{1}}\left[V(t \wedge q)-V_{t}^{E}(t \wedge q)\right]^{2}\right) \\
& \leq 6 T \mathbb{E} \int_{0}^{t \wedge q}\left[\psi^{2}(S(u)-\bar{S}(u))^{2}+\mu^{2}(V(u)-\bar{V}(u))^{2}\right. \\
&\left.+(1-e)^{2} \beta^{2}(V(u) I(u)-\bar{V}(u) \bar{I}(u))^{2}\right] d u \\
&+8(1-e)^{2} \sigma^{2} \mathbb{E} \int_{0}^{t \wedge q}(V(u) I(u)-\bar{V}(u) \bar{I}(u))^{2} d u \\
& \leq 6 \psi^{2} T \mathbb{E} \int_{0}^{t \wedge q}(S(u)-\bar{S}(u))^{2} d u+6 \mu^{2} T \mathbb{E} \int_{0}^{t \wedge q}(V(u)-\bar{V}(u))^{2} d u \\
&+2\left[6(1-e)^{2} \beta^{2} T+8(1-e)^{2} \sigma^{2}\right]\left[\mathbb{E} \int_{0}^{t \wedge q}(V(u) I(u)-\bar{V}(u) I(u))^{2} d u\right. \\
&\left.+\mathbb{E} \int_{0}^{t \wedge q}(\bar{V}(u) I(u)-\bar{V}(u) \bar{I}(u))^{2} d u\right] \\
& \leq h_{1} \mathbb{E} \int_{0}^{t \wedge q}(S(u)-\bar{S}(u))^{2} d u+h_{2} \mathbb{E} \int_{0}^{t \wedge q}(V(u)-\bar{V}(u))^{2} d u \\
&+h_{3} \mathbb{E} \int_{0}^{t \wedge q}(I(u)-\bar{I}(u))^{2} d u \\
& \leq 2 h_{1}\left[\mathbb{E} \int_{0}^{t \wedge q}\left(S(u)-S_{t}^{E}(u)\right)^{2} d u+\mathbb{E} \int_{0}^{t \wedge q}\left(S_{t}^{E}(u)-\bar{S}(u)\right)^{2} d u\right] \\
&+2 h_{2}\left[\mathbb{E} \int_{0}^{t \wedge q}\left(V(u)-V_{t}^{E}(u)\right)^{2} d u+\mathbb{E} \int_{0}^{t \wedge q}\left(V_{t}^{E}(u)-\bar{V}(u)\right)^{2} d u\right]
\end{aligned}
$$




$$
+2 h_{3}\left[\mathbb{E} \int_{0}^{t \wedge q}\left(I(u)-I_{t}^{E}(u)\right)^{2} d u+\mathbb{E} \int_{0}^{t \wedge q}\left(I_{t}^{E}(u)-\bar{I}(u)\right)^{2} d u\right]
$$

where $h_{1}=6 \psi^{2} T, h_{2}=6 \mu^{2} T+12(1-e)^{2} \beta^{2} T K^{2}+16(1-e)^{2} \sigma^{2} K^{2}, h_{3}=12(1-e)^{2} \beta^{2} T K^{2}+$ $16(1-e)^{2} \sigma^{2} K^{2}$.

By Lemma 3.1 plus Eqs. (3.17), (3.19) and (3.21), we have

$$
\begin{aligned}
& \mathbb{E}\left(\sup _{0 \leq t \leq T}\left[\left[S(t \wedge q)-S_{t}^{E}(t \wedge q)\right]^{2}+\left[I(t \wedge q)-I_{t}^{E}(t \wedge q)\right]^{2}+\left[V(t \wedge q)-V_{t}^{E}(t \wedge q)\right]^{2}\right]\right) \\
& \leq H \mathbb{E} \int_{0}^{t \wedge q}\left[\left(S(u)-S_{t}^{E}(u)\right)^{2}+\left(V(u)-V_{t}^{E}(u)\right)^{2}+\left(I(u)-I_{t}^{E}(u)\right)^{2}\right] d u \\
&+C_{1} \Delta^{1-\frac{1}{l}}
\end{aligned}
$$

where $H$ is a positive constant.

An application of the Gronwall inequality will lead to the proof.

Next, we will prove the theorem for the positivity of the balanced method for model (2.8); we can rewrite (2.8) as

$$
\left\{\begin{aligned}
S_{n+1}^{B}= & S_{n}+\left(1+C_{n}\right)^{-1}\left[(1-p) \mu+\alpha \bar{I}_{n}-(\mu+\psi) \bar{S}_{n}-\beta \bar{S}_{n} \bar{I}_{n}\right] \Delta-\left(1+C_{n}\right)^{-1} \sigma \bar{S}_{n} \bar{I}_{n} \Delta w_{n} \\
I_{n+1}^{B}= & I_{n}+\left(1+D_{n}\right)^{-1}\left[\beta \bar{S}_{n} \bar{I}_{n}+(1-e) \beta \bar{V}_{n} \bar{I}_{n}-(\mu+\alpha) \bar{I}_{n}\right] \Delta+\left(1+D_{n}\right)^{-1} \sigma \bar{S}_{n} \bar{I}_{n} \Delta w_{n} \\
& +\left(1+D_{n}\right)^{-1}(1-e) \sigma \bar{V}_{n} \bar{I}_{n} \Delta w_{n}, \\
V_{n+1}^{B}= & V_{n}+\left(1+E_{n}\right)^{-1}\left[p \mu+\psi \bar{S}_{n}-\mu \bar{V}_{n}-(1-e) \beta \bar{V}_{n} \bar{I}_{n}\right] \Delta \\
& -\left(1+E_{n}\right)^{-1}(1-e) \sigma \bar{V}_{n} \bar{I}_{n} \Delta w_{n},
\end{aligned}\right.
$$

when $t \in\left[t_{n}, t_{n+1}\right)$, we define the continuous approximation of the BIM

$$
\left\{\begin{aligned}
S_{n+1}^{B}= & S_{n}+\left(1+C_{n}\right)^{-1}\left[\left[(1-p) \mu+\alpha \bar{I}_{n}-(\mu+\psi) \bar{S}_{n}-\beta \bar{S}_{n} \bar{I}_{n}\right]\left(t-t_{n}\right)\right. \\
& \left.-\sigma \bar{S}_{n} \bar{I}_{n}\left(w(t)-w\left(t_{n}\right)\right)\right] \\
I_{n+1}^{B}= & I_{n}+\left(1+D_{n}\right)^{-1}\left[\left[\beta \bar{S}_{n} \bar{I}_{n}+(1-e) \beta \bar{V}_{n} \bar{I}_{n}-(\mu+\alpha) \bar{I}_{n}\right]\left(t-t_{n}\right)\right. \\
& \left.+\sigma \bar{S}_{n} \bar{I}_{n}\left(w(t)-w\left(t_{n}\right)\right)\right]+\left(1+D_{n}\right)^{-1}(1-e) \sigma \bar{V}_{n} \bar{I}_{n}\left(w(t)-w\left(t_{n}\right)\right) \\
V_{n+1}^{B}= & V_{n}+\left(1+E_{n}\right)^{-1}\left[\left[p \mu+\psi \bar{S}_{n}-\mu \bar{V}_{n}-(1-e) \beta \bar{V}_{n} \bar{I}_{n}\right]\left(t-t_{n}\right)\right. \\
& \left.-(1-e) \sigma \bar{V}_{n} \bar{I}_{n}\left(w(t)-w\left(t_{n}\right)\right)\right] .
\end{aligned}\right.
$$

Thus we can rewritten (3.23) in the integral form as follows:

$$
\left\{\begin{aligned}
S_{t}^{B}= & S_{0}+\int_{0}^{t}\left(1+C_{s}\right)^{-1}\left[(1-p) \mu+\alpha \bar{I}_{s}-(\mu+\psi) \bar{S}_{s}-\beta \bar{S}_{s} \bar{I}_{s}\right] d s \\
& -\int_{0}^{t}\left(1+C_{s}\right)^{-1} \sigma \bar{S}_{s} \bar{I}_{s} d w(s) \\
I_{t}^{B}= & I_{0}+\int_{0}^{t}\left(1+C_{s}\right)^{-1}\left[\beta \bar{S}_{s} \bar{I}_{s}+(1-e) \beta \bar{V}_{s} \bar{I}_{s}-(\mu+\alpha) \bar{I}_{s}\right] d s \\
& +\int_{0}^{t}\left(1+C_{s}\right)^{-1} \sigma \bar{S}_{s} \bar{I}_{s} d w(s)+\int_{0}^{t}\left(1+C_{s}\right)^{-1}(1-e) \sigma \bar{V}_{s} \bar{I}_{s} d w(s) \\
V_{t}^{B}= & V_{0}+\int_{0}^{t}\left(1+C_{s}\right)^{-1}\left[p \mu+\psi \bar{S}_{s}-\mu \bar{V}_{s}-(1-e) \beta \bar{V}_{s} \bar{I}_{s}\right] d s \\
& -\int_{0}^{t}\left(1+C_{s}\right)^{-1}(1-e) \sigma \bar{V}_{s} \bar{I}_{s} d w(s) .
\end{aligned}\right.
$$

In order to estimate if the BIM approximation solution will converge to the true solution, we plan to prove a strong convergence result. 
Theorem 3.2 For any constant $C_{3}$ and integer $l \geq 2$, the BIM (2.8) converge to the true solution of model (1.1) with strong order $\frac{1}{2}\left(1-\frac{1}{l}\right)$, i.e., such that

$$
\mathbb{E}\left(\sup _{0 \leq t \leq T}\left[\left[S_{t}-S_{t}^{B}\right]^{2}+\left[I_{t}-I_{t}^{B}\right]^{2}+\left[V_{t}-V_{t}^{B}\right]^{2}\right]\right) \leq C_{3} \Delta^{1-\frac{1}{l}}
$$

Proof By Theorem 3.1, telling us that the Euler solution is convergent with order $\left(1-\frac{1}{l}\right)$ in the mean square sense, for $t \in T$, we have

$$
\begin{aligned}
& \mathbb{E}\left(\sup _{0 \leq t \leq T}\left[\left[S_{t}-S_{t}^{B}\right]^{2}+\left[I_{t}-I_{t}^{B}\right]^{2}+\left[V_{t}-V_{t}^{B}\right]^{2}\right]\right) \\
&=\mathbb{E}\left(\operatorname { s u p } _ { 0 \leq t \leq T } \left[\left(S_{t}-S_{t}^{E}+S_{t}^{E}-S_{t}^{B}\right)^{2}+\left(I_{t}-I_{t}^{E}+I_{t}^{E}-I_{t}^{B}\right)^{2}\right.\right. \\
&\left.\left.\quad+\left(V_{t}-V_{t}^{E}+V_{t}^{E}-V_{t}^{B}\right)^{2}\right]\right) \\
& \leq 2 \mathbb{E}\left(\operatorname { s u p } _ { 0 \leq t \leq T } \left[\left(S_{t}-S_{t}^{E}\right)^{2}+\left(S_{t}^{E}-S_{t}^{B}\right)^{2}+\left(I_{t}-I_{t}^{E}\right)^{2}+\left(I_{t}^{E}-I_{t}^{B}\right)^{2}\right.\right. \\
&\left.\left.\quad+\left(V_{t}-V_{t}^{E}\right)^{2}+\left(V_{t}^{E}-V_{t}^{B}\right)^{2}\right]\right) \\
& \leq 2 \mathbb{E}\left(\sup _{0 \leq t \leq T}\left[\left(S_{t}-S_{t}^{E}\right)^{2}+\left(I_{t}-I_{t}^{E}\right)^{2}+\left(V_{t}-V_{t}^{E}\right)^{2}\right]\right) \\
& \quad+2 \mathbb{E}\left(\sup _{0 \leq t \leq T}\left[\left(S_{t}^{E}-S_{t}^{B}\right)^{2}+\left(I_{t}^{E}-I_{t}^{B}\right)^{2}+\left(V_{t}-V_{t}^{E}\right)^{2}\right]\right) \\
& \leq C_{1} \Delta^{1-\frac{1}{l}}+2 \mathbb{E}\left(\sup _{0 \leq t \leq T}\left[\left(S_{t}^{E}-S_{t}^{B}\right)^{2}+\left(I_{t}^{E}-I_{t}^{B}\right)^{2}+\left(V_{t}^{E}-V_{t}^{B}\right)^{2}\right]\right) .
\end{aligned}
$$

Now, we estimate the terms of Eq. (3.26).

First, for $\left(S_{t}^{E}-S_{t}^{B}\right)^{2}$, we have

$$
\begin{aligned}
\left(S_{t}^{E}-S_{t}^{B}\right)^{2}= & \left(\int_{0}^{t}\left[1-\left(1+C_{s}\right)^{-1}\right]\left[(1-p) \mu+\alpha \bar{I}_{s}-(\mu+\psi) \bar{S}_{s}-\beta \bar{S}_{s} \bar{I}_{s}\right] d s\right. \\
& \left.-\int_{0}^{t}\left[1-\left(1+C_{s}\right)^{-1}\right] \sigma \bar{S}_{s} \bar{I}_{s} d w(s)\right)^{2},
\end{aligned}
$$

we hence have

$$
\begin{aligned}
& \mathbb{E}\left[\sup _{0 \leq t \leq T}\left(S_{t}^{E}-S_{t}^{B}\right)^{2}\right] \\
& \leq 8 T \mathbb{E} \int_{0}^{T}\left[1-\left(1+C_{s}\right)^{-1}\right]^{2}\left[(1-p)^{2} \mu^{2}+\alpha^{2} \bar{I}_{s}^{2}+(\mu+\psi)^{2} \bar{S}_{s}^{2}+\beta^{2} \bar{S}_{s}^{2} \bar{I}_{s}^{2}\right] d s \\
&+8 \mathbb{E} \int_{0}^{T}\left[1-\left(1+C_{s}\right)^{-1}\right]^{2} \sigma^{2} \bar{S}_{s}^{2} \bar{I}_{s}^{2} d s \\
& \leq 8 T \int_{0}^{T} \mathbb{E}\left(C_{s}\left(1+C_{s}\right)^{-1}\right)^{2}\left[(1-p)^{2} \mu^{2}+\alpha^{2} \bar{I}_{s}^{2}+(\mu+\psi)^{2} \bar{S}_{s}^{2}+\beta^{2} \bar{S}_{s}^{2} \bar{I}_{s}^{2}\right] d s \\
&+8 \int_{0}^{T} \mathbb{E}\left(C_{s}\left(1+C_{s}\right)^{-1}\right)^{2} \sigma^{2} \bar{S}_{s}^{2} \bar{I}_{s}^{2} d s \\
& \leq 8 M^{2} T \int_{0}^{T} \mathbb{E}\left(C_{s}\right)^{2}\left[(1-p)^{2} \mu^{2}+\alpha^{2} \bar{I}_{s}^{2}+(\mu+\psi)^{2} \bar{S}_{s}^{2}+\beta^{2} \bar{S}_{s}^{2} \bar{I}_{s}^{2}\right] d s \\
&+8 M^{2} \int_{0}^{T} \mathbb{E}\left(C_{s}\right)^{2} \sigma^{2} \bar{S}_{s}^{2} \bar{I}_{s}^{2} d s .
\end{aligned}
$$


Noticing that the control functions $\left(\mu+\psi+\beta I_{n}\right)$ and $\sigma I_{n}$ are bounded, that is to say, there exists a positive constant $A_{1}$ such that $\mathbb{E}\left(C_{s}\right)^{2} \leq A_{1}\left(\Delta^{2}+\Delta\right)$, where $A_{1}=\max \left\{6\left(\mu^{2}+\psi^{2}+\right.\right.$ $\left.\beta^{2} K^{2}\right), 2 \sigma^{2} K^{2}$, we can get

$$
\begin{aligned}
\mathbb{E}\left[\sup _{0 \leq t \leq T}\left(S_{t}^{E}-S_{t}^{B}\right)^{2}\right] \leq & 8 M^{2} T\left((1-p)^{2} \mu^{2}+\alpha^{2} K^{2}+(\mu+\psi)^{2} K^{2}+\beta^{2} K^{4}\right) A_{1}\left(\Delta^{2}+\Delta\right) \\
& +8 M^{2} K^{4} \sigma^{2} A_{1}\left(\Delta^{2}+\Delta\right) \\
\leq & C_{4} \Delta
\end{aligned}
$$

where $C_{4}=16 M^{2} T\left((1-p)^{2} \mu^{2}+\alpha^{2} K^{2}+(\mu+\psi)^{2} K^{2}+\beta^{2} K^{4}\right) A_{1}+16 M^{2} K^{4} \sigma^{2} A_{1}$.

Next, for $\left(I_{t}^{E}-I_{t}^{B}\right)^{2}$, we have

$$
\begin{aligned}
\left(I_{t}^{E}-\right. & \left.I_{t}^{B}\right)^{2} \\
= & \left(\int_{0}^{t}\left[1-\left(1+D_{s}\right)^{-1}\right]\left[\beta \bar{S}_{s} \bar{I}_{s}+(1-e) \beta \bar{V}_{s} \bar{I}_{s}-(\mu+\alpha) \bar{I}_{s}\right] d s\right. \\
& +\int_{0}^{t}\left[1-\left(1+C_{s}\right)^{-1}\right] \sigma \bar{S}_{s} \bar{I}_{s} d w(s) \\
& \left.+\int_{0}^{t}\left[1-\left(1+D_{s}\right)^{-1}\right](1-e) \sigma \bar{V}_{s} \bar{I}_{s} d w(s)\right)^{2},
\end{aligned}
$$

we hence have

$$
\begin{aligned}
& \mathbb{E}\left[\sup _{0 \leq t \leq T}\left(I_{t}^{E}-I_{t}^{B}\right)^{2}\right] \\
& \leq 9 T \mathbb{E} \int_{0}^{t}\left[1-\left(1+D_{s}\right)^{-1}\right]^{2}\left[\beta^{2} \bar{S}_{s}^{2} \bar{I}_{s}^{2}+(1-e)^{2} \beta^{2} \bar{V}_{s}^{2} \bar{I}_{s}^{2}+(\mu+\alpha)^{2} \bar{I}_{s}^{2}\right] d s \\
&+12 \mathbb{E} \int_{0}^{t}\left[1-\left(1+D_{s}\right)^{-1}\right]^{2} \sigma^{2} \bar{S}_{s}^{2} \bar{I}_{s}^{2} d s+12 \mathbb{E} \int_{0}^{t}\left[1-\left(1+D_{s}\right)^{-1}\right]^{2}(1-e)^{2} \sigma^{2} \bar{V}_{s}^{2} \bar{I}_{s}^{2} d s \\
& \leq 9 T \int_{0}^{t} \mathbb{E}\left(D_{s}\left(1+D_{s}\right)^{-1}\right)^{2}\left[\beta^{2} \bar{S}_{s}^{2} \bar{I}_{s}^{2}+(1-e)^{2} \beta^{2} \bar{V}_{s}^{2} \bar{I}_{s}^{2}+(\mu+\alpha)^{2} \bar{I}_{s}^{2}\right] d s \\
&+12 \int_{0}^{t} \mathbb{E}\left(D_{s}\left(1+D_{s}\right)^{-1}\right)^{2} \sigma^{2} \bar{S}_{s}^{2} \bar{I}_{s}^{2} d s+12 \int_{0}^{t} \mathbb{E}\left(D_{s}\left(1+D_{s}\right)^{-1}\right)^{2}(1-e)^{2} \sigma^{2} \bar{V}_{s}^{2} \bar{I}_{s}^{2} d s \\
& \leq 9 T M^{2} \int_{0}^{t} \mathbb{E}\left(D_{s}\right)^{2}\left[\beta^{2} \bar{S}_{s}^{2} \bar{I}_{s}^{2}+(1-e)^{2} \beta^{2} \bar{V}_{s}^{2} \bar{I}_{s}^{2}+(\mu+\alpha)^{2} \bar{I}_{s}^{2}\right] d s \\
&+12 M^{2} \int_{0}^{t} \mathbb{E}\left(D_{s}\right)^{2} \sigma^{2} \bar{S}_{s}^{2} \bar{I}_{s}^{2} d s+12 M^{2} \int_{0}^{t} \mathbb{E}\left(D_{s}\right)^{2}(1-e)^{2} \sigma^{2} \bar{V}_{s}^{2} \bar{I}_{s}^{2} d s .
\end{aligned}
$$

Noticing that the control functions $(\mu+\alpha)$ and $\sigma\left(S_{n}+V_{n}\right)$ are bounded, that is to say, there exists a positive constant $A_{2}$ such that $\mathbb{E}\left(D_{s}\right)^{2} \leq A_{2}\left(\Delta^{2}+\Delta\right)$, where $A_{2}=\max \left\{4\left(\mu^{2}+\right.\right.$ $\left.\left.\alpha^{2}\right), 8 \sigma^{2} K^{2}\right\}$, we can get

$$
\begin{aligned}
\mathbb{E}\left[\sup _{0 \leq t \leq T}\left(I_{t}^{E}-I_{t}^{B}\right)^{2}\right] \leq & 9 T M^{2}\left[\beta^{2} K^{4}+(1-e)^{2} \beta^{2} K^{4}+(\mu+\alpha)^{2} K^{2}\right] A_{2}\left(\Delta^{2}+\Delta\right) \\
& +12 M^{2}\left[\sigma^{2} K^{4}+(1-e)^{2} \sigma^{2} K^{4}\right] A_{2}\left(\Delta^{2}+\Delta\right) \\
\leq & C_{5} \Delta,
\end{aligned}
$$


where $C_{5}=18 T M^{2}\left[\beta^{2} K^{4}+(1-e)^{2} \beta^{2} K^{4}+(\mu+\alpha)^{2} K^{2}\right] A_{2}+24 M^{2}\left[\sigma^{2} K^{4}+(1-e)^{2} \sigma^{2} K^{4}\right] A_{2}$. And then, for $\left(V_{t}^{E}-V_{t}^{B}\right)^{2}$, we have

$$
\begin{aligned}
\left(V_{t}^{E}-V_{t}^{B}\right)^{2}= & \left(\int_{0}^{t}\left[1-\left(1+E_{s}\right)^{-1}\right]\left[p \mu+\psi \bar{S}_{s}-\mu \bar{V}_{s}-(1-e) \beta \bar{V}_{s} \bar{I}_{s}\right] d s\right. \\
& \left.-\int_{0}^{t}\left[1-\left(1+E_{s}\right)^{-1}\right](1-e) \sigma \bar{V}_{s} \bar{I}_{s} d w(s)\right)^{2}
\end{aligned}
$$

we hence have

$$
\begin{aligned}
& \mathbb{E}\left[\sup _{0 \leq t \leq T}\left(V_{t}^{E}-V_{t}^{B}\right)^{2}\right] \\
& \leq 8 T \mathbb{E} \int_{0}^{t}\left[1-\left(1+E_{s}\right)^{-1}\right]^{2}\left[p^{2} \mu^{2}+\psi^{2} \bar{S}_{s}^{2}+\mu^{2} \bar{V}_{s}^{2}+(1-e)^{2} \beta^{2} \bar{V}_{s}^{2} \bar{I}_{s}^{2}\right] d s \\
&+8 \mathbb{E} \int_{0}^{t}\left[1-\left(1+E_{s}\right)^{-1}\right]^{2}(1-e)^{2} \sigma^{2} \bar{V}_{s}^{2} \bar{I}_{s}^{2} d s \\
& \leq 8 T \int_{0}^{t} \mathbb{E}\left(E_{s}\left(1+E_{s}\right)^{-1}\right)^{2}\left[p^{2} \mu^{2}+\psi^{2} \bar{S}_{s}^{2}+\mu^{2} \bar{V}_{s}^{2}+(1-e)^{2} \beta^{2} \bar{V}_{s}^{2} \bar{I}_{s}^{2}\right] d s \\
&+8 \int_{0}^{t} \mathbb{E}\left(E_{s}\left(1+E_{s}\right)^{-1}\right)^{2}(1-e)^{2} \sigma^{2} \bar{V}_{s}^{2} \bar{I}_{s}^{2} d s \\
& \leq 8 T M^{2} \int_{0}^{t} \mathbb{E}\left(E_{s}\right)^{2}\left[p^{2} \mu^{2}+\psi^{2} \bar{S}_{s}^{2}+\mu^{2} \bar{V}_{s}^{2}+(1-e)^{2} \beta^{2} \bar{V}_{s}^{2} \bar{I}_{s}^{2}\right] d s \\
&+8 M^{2} \int_{0}^{t} \mathbb{E}\left(E_{s}\right)^{2}(1-e)^{2} \sigma^{2} \bar{V}_{s}^{2} \bar{I}_{s}^{2} d s .
\end{aligned}
$$

Noticing that the control functions $\left(\mu+\beta I_{n}\right)$ and $\sigma I_{n}$ are bounded, that is to say, there exists a positive constant $A_{3}$ such that $\mathbb{E}\left(E_{s}\right)^{2} \leq A_{3}\left(\Delta^{2}+\Delta\right)$, where $A_{3}=\max \left\{4\left(\mu^{2}+\right.\right.$ $\left.\left.\beta^{2} K^{2}\right), 2 \sigma^{2} K^{2}\right\}$, we can get

$$
\begin{aligned}
\mathbb{E}\left[\sup _{0 \leq t \leq T}\left(V_{t}^{E}-V_{t}^{B}\right)^{2}\right] \leq & 8 T M^{2}\left[p^{2} \mu^{2}+\psi^{2} K^{2}+\mu^{2} K^{2}+(1-e)^{2} \beta^{2} K^{4}\right] A_{3}\left(\Delta^{2}+\Delta\right) \\
& +8 M^{2}(1-e)^{2} \sigma^{2} K^{4} A_{3}\left(\Delta^{2}+\Delta\right) \\
\leq & C_{6} \Delta
\end{aligned}
$$

where $C_{6}=16 T M^{2}\left[p^{2} \mu^{2}+\psi^{2} K^{2}+\mu^{2} K^{2}+(1-e)^{2} \beta^{2} K^{4}\right] A_{3}+16 M^{2}(1-e)^{2} \sigma^{2} K^{4} A_{3}$.

Thus, taking (3.28), (3.31) and (3.34) into (3.26), we can obtain

$$
\begin{aligned}
\mathbb{E}\left(\sup _{0 \leq t \leq T}\left[\left[S_{t}-S_{t}^{B}\right]^{2}+\left[I_{t}-I_{t}^{B}\right]^{2}+\left[V_{t}-V_{t}^{B}\right]^{2}\right]\right) & \leq C_{1} \Delta^{1-\frac{1}{l}}+C_{6} \Delta \\
& \leq C_{3} \Delta^{1-\frac{1}{l}}
\end{aligned}
$$

The proof is completed.

Remark 3.1 Theorem 3.2 shows that $S(t), I(t), V(t)$ be the true solution and $S_{t}^{B}, I_{t}^{B}$, $V_{t}^{B}$ be the BIM approximate solution are close to each other in the sense, and the BIM converges to the true solution of stochastic SIVS epidemic model with order $\frac{1}{2}\left(1-\frac{1}{l}\right)$. 


\section{Numerical experiments}

In this section, we will give one example to illustrate the theoretical results. Let us consider the following stochastic SIVS epidemic model with scalar Brownian motion.

$$
\left\{\begin{array}{l}
d S=[(1-p) \mu+\alpha I-(\mu+\psi) S-\beta S I] d t-\sigma S I d w(t), \\
d I=[\beta S I+(1-e) \beta V I-(\mu+\alpha) I] d t+\sigma S I d w(t)+(1-e) \sigma V I d w(t), \\
d V=[p \mu+\psi S-\mu V-(1-e) \beta V I] d t-(1-e) \sigma V I d w(t),
\end{array}\right.
$$

where initial data $(S(0), I(0), V(0))=(0.8,0.1,0.2)$, with parameter values $p=0.8, \beta=0.65$, $\mu=0.43, \alpha=0.2, \psi=0.32, e=0.2$ and $\sigma=1.3$.

Now, we resort MATLAB to investigate the numerical approximation solution of EM scheme and BIM scheme for the stochastic epidemic model. And fixing $T=100, \Delta=0.05$. We choose the control function $C_{k}=\left(\beta I_{k}+\mu+\psi\right) \Delta+\sigma I_{k}\left|\Delta w_{k}\right|, D_{k}=(\mu+\alpha) \Delta+\sigma\left(S_{k}+\right.$ $\left.V_{k}\right)\left|\Delta w_{k}\right|$ and $E_{k}=\left(\beta I_{k}+\mu\right) \Delta+\sigma I_{k}\left|\Delta w_{k}\right|$. Then we compare the BIM and EM method in Fig. 1.

Figure 1 draws the numerical solution obtained from BIM and EM method. We present results for three different variables for the SIVS model. Figure 1(a) delineates the path of the EM solution and BIM numerical solution for $S(t)$. Figure 1(b) depicts the sample paths of the EM solution and BIM numerical solution for $I(t)$. Figure 1(c) describes the sample paths of the EM solution and BIM numerical solution for $V(t)$. Through the comparison between EM and BIM method, we clearly that the numerical solution of BIM can preserve positivity, and the EM path becomes negative. That is to say, the EM method cannot maintain the positivity of Fig. 1.

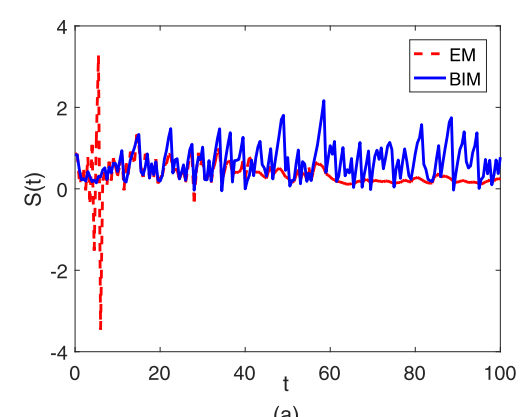

(a)

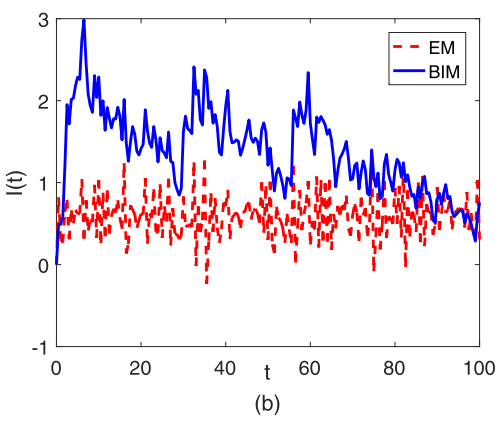

(b)

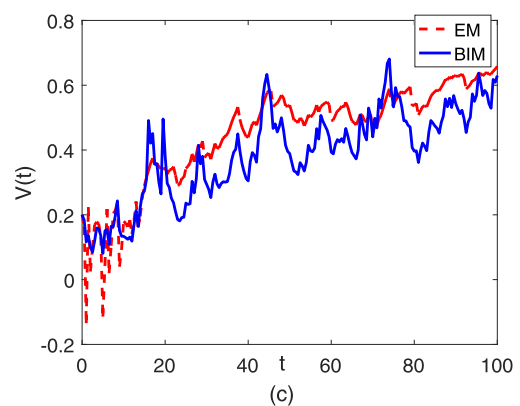

Figure 1 The EM and BIM method numerical simulations of solution $S(t), I(t), V(t)$ for model (1.1) 
Table 1 Percentage of negative paths for the EM scheme and BIM

\begin{tabular}{|c|c|c|c|c|c|c|c|c|c|}
\hline \multirow[t]{2}{*}{ Number } & \multicolumn{3}{|l|}{ S } & \multicolumn{3}{|l|}{1} & \multicolumn{3}{|l|}{$V$} \\
\hline & $\Delta=0.1$ & $\Delta=0.05$ & $\Delta=0.01$ & $\Delta=0.1$ & $\Delta=0.05$ & $\Delta=0.01$ & $\overline{\Delta=0.1}$ & $\Delta=0.05$ & $\Delta=0.01$ \\
\hline $\begin{array}{l}E M \\
B I M\end{array}$ & $\begin{array}{l}33.15 \% \\
0 \%\end{array}$ & $\begin{array}{l}28.67 \% \\
0 \%\end{array}$ & $\begin{array}{l}13.59 \% \\
0 \%\end{array}$ & $\begin{array}{l}32.55 \% \\
0 \%\end{array}$ & $\begin{array}{l}20.77 \% \\
0 \%\end{array}$ & $\begin{array}{l}15.35 \% \\
0 \%\end{array}$ & $\begin{array}{l}20.68 \% \\
0 \%\end{array}$ & $\begin{array}{l}15.56 \% \\
0 \%\end{array}$ & $\begin{array}{l}12.56 \% \\
0 \%\end{array}$ \\
\hline
\end{tabular}

In Table 1, we propose the percentage of negative paths simulating the stochastic SIVS epidemic model (1.1). Just as the theoretical results, by averaging overall of the 1000 samples, we found that the EM method have negative values on a certain probability of susceptible $S$, infected $I$ and vaccinated $V$, respectively. But the BIM can preserve positivity for all step sizes in $[0, T]$. This analysis may offer us some advise when we are faced with epidemic diseases.

\section{Conclusions}

The positivity-preserving numerical method for the stochastic SIVS epidemic model has been systematically discussed in this paper. First, we propose a SIVS epidemic model with vaccination and investigate the EM numerical approximate solution to the model (1.1). Meanwhile, we prove the convergence property of EM approximate solution to the true solution. Then we establish the balanced implicit method for the stochastic SIVS model. Preserving positivity of the proposed method is proved. Numerical results reveal that the BIM is verifying the availability of the approach for maintaining positivity.

Another interesting topic should be further conducted to reveal how to construct a numerical method preserving positivity for stochastic age-structured SIVS epidemic model. We regard that as our future work.

\section{Acknowledgements \\ The authors would like to express their sincere thanks to referees and the editor for their enthusiastic guidance and help. \\ Funding \\ The research was funded by the 'Major Innovation Projects for Building First-class Universities in China's Western Region' (ZKZD2017009).}

Competing interests

The authors declare that they have no competing interests.

Authors' contributions

All authors read and approved the final manuscript.

\section{Publisher's Note}

Springer Nature remains neutral with regard to jurisdictional claims in published maps and institutional affiliations.

Received: 8 October 2018 Accepted: 15 January 2019 Published online: 24 January 2019

\section{References}

1. Shi, R., Jiang, X., Chen, L.: The effect of impulsive vaccination on an SIR epidemic model. Appl. Math. Comput. 212, 305-311 (2009)

2. Nie, L., Shen, J., Yang, C.: Dynamic behavior analysis of SIVS epidemic models with state-dependent pulse vaccination. Nonlinear Anal. Hybrid Syst. 27, 258-270 (2018)

3. Liu, L., Wang, J., Liu, X.: Global stability of an SEIR epidemic model with age-dependent latency and relapse. Nonlinear Anal. 24, 18-35 (2015)

4. Omondi, O., Wang, C., Xue, X., Lawi, O.: Modeling the effects of vaccination on rotavirus infection. Adv. Differ. Equ. 2015, $381(2015)$

5. Lin, Y., Jiang, D., Wang, S.: Stationary distribution of a stochastic SIS epidemic model with vaccination. Physica A 394 187-197 (2014)

6. Tornatore, E., Vetro, P., Buccellato, S.M.: SVIR epidemic model with stochastic perturbation. Neural Comput. Appl. 24, 309-315 (2014) 
7. Zhao, Y., Jiang, D., O'Regan, D.: The extinction and persistence of the stochastic SIS epidemic model with vaccination. Physica A 392, 4916-4927 (2013)

8. Lu, R., Wei, F.: Persistence and extinction for an age-structured stochastic SVIR epidemic model with generalized nonlinear incidence rate. Physica A 513, 572-587 (2019)

9. Yang, J., Martchev, M., Wang, L.: Global threshold dynamics of an SIVS model with waning vaccine-induced immunity and nonlinear incidence. Math. Biosci. 268, 1-8 (2015)

10. Zhao, Y., Jiang, D.: The threshold of a stochastic SIRS epidemic model with saturated incidence. Appl. Math. Lett. 34, 90-93 (2014)

11. Wen, B., Teng, Z., Li, Z.: The threshold of a periodic stochastic SIVS epidemic model with nonlinear incidence. Physica A 508, 532-549 (2018)

12. Mao, X.: The truncated Euler-Maruyama method for stochastic differential equations. J. Comput. Appl. Math. 290, 370-384 (2015)

13. Hu, Y: Semi-implicit Euler-Maruyama scheme for stiff stochastic equations. In: Stochastic Analysis and Related Topics V, vol. 38, pp. 183-202 (1996)

14. Milošević, M.: The Euler-Maruyama approximation of solutions to stochastic differential equations with piecewise constant arguments. J. Comput. Appl. Math. 298, 1-12 (2016)

15. Bayram, M., Partal, T., Buyukoz, G.: Numerical methods for simulation of stochastic differential equations. Adv. Differ. Equ. 2018, 17 (2018)

16. Higham, D., Mao, X., Szpruch, L.: Convergence, non-negativity and stability of a new Milstein scheme with applications to finance. Discrete Contin. Dyn. Syst. 8, 2083-2100 (2017)

17. Kahl, C., Gunther, M., Rossberg, T.: Structure preserving stochastic integration schemes in interest rate derivative modeling. Appl. Numer. Math. 58, 284-295 (2008)

18. Milstein, G., Platen, E., Schurz, H.: Balanced implicit methods for stiff stochastic systems. SIAM J. Numer. Anal. 35, $1010-1019(1998)$

19. Tan, J., Men, W., Pei, Y., Guo, Y.: Construction of positivity preserving numerical method for stochastic age-dependent population equations. Appl. Math. Comput. 293, 57-64 (2017)

20. Liu, Q., Jiang, D., Shi, N., et al.: The threshold of a stochastic SIS epidemic model with imperfect vaccination. Math. Comput. Simul. 144, 78-90 (2018)

21. Schurz, H.: Numerical regularization for SDEs: construction of nonnegative solutions. Dyn. Syst. Appl. 5, 323-352 (1996)

22. Mao, X.: Stochasti Differential Equations and Their Application. Horwood Publishing Series in Mathematics and Applications. Horwood, Chichester (1997)

\section{Submit your manuscript to a SpringerOpen ${ }^{\circ}$ journal and benefit from:}

- Convenient online submission

- Rigorous peer review

- Open access: articles freely available online

- High visibility within the field

- Retaining the copyright to your article

Submit your next manuscript at $\gg$ springeropen.com 